\title{
Acute Mesenteric Ischemia
}

\author{
Florian Kühn $^{a}$ Tobias S. Schiergens ${ }^{a}$ Ernst Klar ${ }^{b}$ \\ aDepartment of General, Visceral, and Transplantation Surgery, Ludwig Maximilian University of Munich, Munich, \\ Germany; ${ }^{b}$ Department of General, Visceral, Vascular and Transplantation Surgery, University Medical Center \\ Rostock, Rostock, Germany
}

\begin{abstract}
Keywords
Acute mesenteric ischemia . Portal vein thrombosis .

Diagnosis · Diagnostic imaging · Endovascular procedures ·

Surgery $\cdot$ Critical care $\cdot$ Mortality
\end{abstract}

\begin{abstract}
Background: Despite constant improvements in diagnostic as well as interventional and surgical techniques, acute mesenteric ischemia (AMI) remains a life-threatening emergency with high mortality rates. The time to diagnosis of AMI is the most important predictor of patients' outcome; therefore, prompt diagnosis and intervention are essential to reduce mortality in patients with AMI. The present review was performed to analyze potential risk factors and to help find ways to improve the outcome of patients with AMI. Summary: Whereas AMI only applies to approximately $1 \%$ of all patients with an "acute abdomen," its incidence is rising up to $10 \%$ in patients $>70$ years of age. The initial clinical stage of $\mathrm{AMI}$ is characterized by a sudden onset of strong abdominal pain followed by a painless interval. Depending on the extent of disease, the symptoms of nonocclusive mesenteric ischemia (NOMI) and patients with a venous thrombosis can be very different from those of acute occlusive ischemia. $\mathrm{Bi}$ phasic contrast-enhanced CT represents the gold standard for the diagnosis of arterial and venous occlusion. In case of a central occlusion of the superior mesenteric artery or signs of peritonitis, immediate surgery should be performed. If major bowel resection becomes necessary, critical residual intestinal length limits must be kept in mind. Endovascular techniques for arterial occlusion have taken on a much
\end{abstract}

greater importance today. For stable patients with NOMI, interventional catheter angiography is recommended because it enables diagnosis and treatment with selective application of vasodilators. Depending on its degree, interventional treatment with a transhepatic catheter lysis should be considered for acute and chronic portal vein thrombosis. Key Message: The prompt and targeted use of the appropriate diagnostics and interventions appears to be the only way to reduce the persistently high mortality rates for AMI.

(c) 2020 S. Karger AG, Basel

\section{Introduction}

Acute mesenteric ischemia (AMI) is a vascular emergency that is caused by an interruption of the blood supply to the small intestine. Despite constant improvements in diagnostic, interventional and surgical techniques, AMI remains a life-threatening emergency with high mortality rates ranging between 50 and $70 \%$ [1-5]. The time to diagnosis of AMI is the most important predictor of patients' outcome; therefore, prompt diagnosis and intervention are essential to reduce mortality in patients with AMI. The present review was performed to analyze potential risk factors and to help find ways to improve the outcome of patients with AMI. In the following review, the different forms of mesenteric ischemia, namely acute occlusive mesenteric ischemia, non-occlusive mesenteric ischemia (NOMI), and venous thrombosis of the mesenteric-portal axis, will be discussed in detail. 
Fig. 1. Clinical manifestation and course of AMI with loss of time due to routine diagnostics. With kind permission from Georg Thieme Verlag KG, Stuttgart, Germany modified from [2].

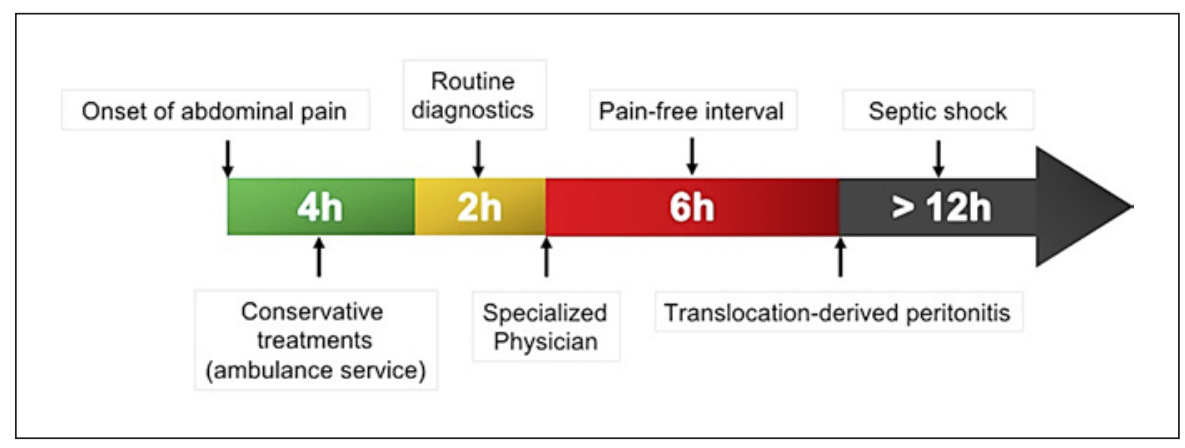

\section{Occlusive Mesenteric Ischemia}

\section{Definition and Epidemiology}

AMI refers to the sudden onset of small intestinal hypoperfusion due to factors such as mesenteric arterial embolism (50\%), mesenteric arterial thrombosis (15-25\%), or mesenteric venous thrombosis $(5-15 \%)[4,5]$. Whereas AMI only applies to approximately $1 \%$ of all patients with an "acute abdomen," its incidence is significantly rising in patients $>70$ years of age [1]. Several risk factors have been reported, among them heart failure, atrial fibrillation, coronary heart disease, arterial hypertension, and peripheral vascular disease should be considered for AMI [1-5]. AMI is a time-critical emergency resulting in irreversible hypoperfusion of the mesenteric organs within a few hours, leading to a high mortality rate.

\section{Anatomy and Pathophysiology}

The superior mesenteric artery (SMA) is a single central vessel with a vulnerable terminal vascular zone. In around $85 \%$ of the cases, the functional terminal vascular zone of the SMA that runs from the central collateral blood supply to the mobile convolutions of the small intestine is affected $[1,2]$. The large diameter and narrow take-off angle of the SMA contribute to its anatomical susceptibility to embolism. Due to collateralization, the coeliac and inferior mesenteric arteries are phylogenetically better protected against an acute occlusion of their main trunks. The initial clinical stage of AMI is characterized by the sudden onset of strong, spasmodic abdominal pain; after 3-6 h, this phase is followed by a painless interval due to the damage of the intramural pain receptors as a result of prolonged hypoperfusion [1]. An acute complete circulatory disruption of the intestine leads to irreversible mucosal ischemia with leukocyte infiltration and

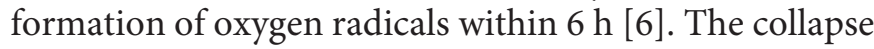
of the mucosal barrier further contributes to bacterial translocation and gangrene of the intestinal wall. In addition, bacterial infiltration leads to peritonitis, ileus, sepsis, and multiorgan failure [1]. The mortality rate rises from 0 to $10 \%$ in cases with immediate treatment, to $50 \%$ in

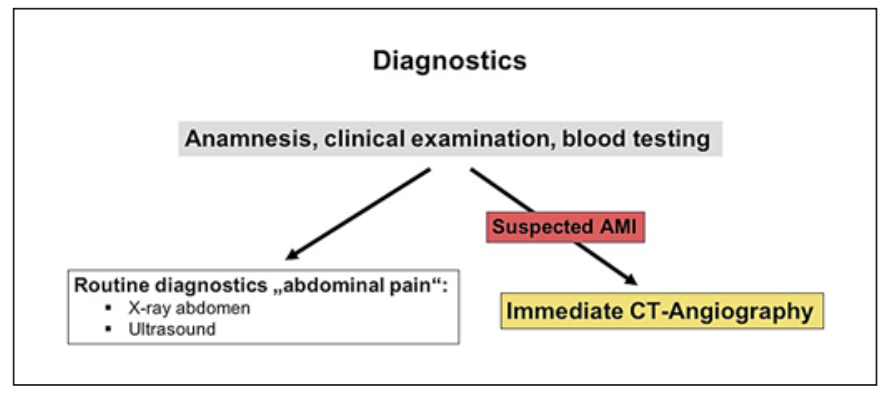

Fig. 2. Diagnostic algorithm in case of unspecific abdominal pain versus suspected AMI.

those with delays of $6-12 \mathrm{~h}$, and $80-100 \%$ with delays of more than $24 \mathrm{~h}$ after the onset of symptoms [7]. Figure 1 illustrates the timeline from the onset of symptoms until irreversible gangrene and multi-organ failure

\section{Diagnostics}

Time-saving and focused diagnostics are vital in detecting an AMI as early as possible. An unspecific routine diagnostic program or the wrong diagnostic tools extend the time to diagnosis and contribute to the high mortality of AMI. The diagnostic delay is $8 \mathrm{~h}$ on average, and therapy alone requires at least another mean of $2.5 \mathrm{~h}$ before reperfusion of the mesenteric region is achieved $[1,2]$. Already within a warm ischemia time of $6 \mathrm{~h}$, there is disintegration of the mucosal barrier with subsequent bacterial translocation and morphological changes of the intestinal wall. Therefore, a focused anamnesis, the recognition of predisposing factors, and a precise clinical examination are the key to time-saving diagnostics and improved survival in patients with AMI. If there is any suspicion of acute occlusive mesenteric ischemia, biphasic contrast-enhanced computed tomography with threedimensional multiplanar reconstruction computed tomography $(\mathrm{CT})$ is the diagnostic tool of choice $[1,2]$. Figure 2 illustrates the diagnostic approach in case of unspecific abdominal pain and the preferred algorithm for patients suspicious of AMI. 

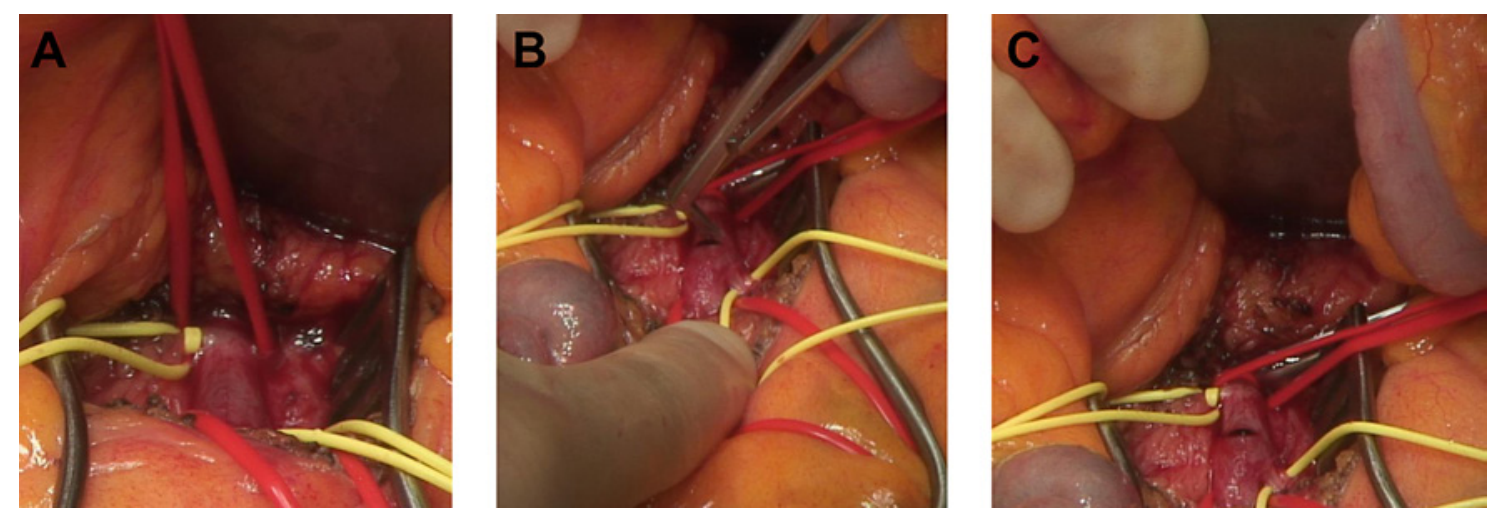

Fig. 3. A Intraoperative exposition of the SMA, occluded with an embolus in a patient with AMI. B, C Incision of the SMA prior to embolectomy.

The CT scan should include the whole abdomen in both the arterial and venous phases [1]. The venous phase is required for the diagnosis of mesenteric venous thrombosis. In this protocol, there is no need for oral contrast agents because they do not improve the imaging of intestinal wall alterations but rather contribute to the loss of time during diagnostics. It is of fundamental importance to communicate the clinical suspicion of AMI with the radiologist when requesting the diagnostic imaging. Although magnetic resonance imaging can theoretically be used for the diagnosis of AMI, CT should be preferred to safe time. The universal availability and the quality of multidetector CT allows quick and sufficient enhancement of the mesenteric vessels with further three-dimensional reconstruction by CT angiography to accurately depict the mesenteric vascular anatomy. In addition to detailed imaging of the intestinal wall, the main advantages of CT angiography over catheter angiography is the ability to rule out other differential diagnoses of AMI. The sensitivity and specificity of multiplanar reconstruction CT are 93 and 100\%, respectively; its positive and negative predictive values are between 94 and $100 \%[8,9]$. Consequently, the use of catheter angiography is becoming less popular [10]. Because mesenteric ischemia generally leads to a distension of intestinal loops, ultrasound should not be primarily used for examination (Class III recommendation, level of evidence $\mathrm{C}$, according to ACC/ AHA guidelines). The widespread use of conventional abdominal film does not lead to a significant time delay; next to a possible exclusion of free abdominal air it can also show signs of an advanced intestinal ischemia such as air bubbles within the intestinal wall (pneumatosis intestinalis).

\section{Laboratory Parameters}

Various serum markers are thought to be associated with AMI; however, none of the parameters are equipped with a sufficiently high sensitivity or specificity for the diagnosis of AMI [1]. Serum lactate serves as a non-specific parameter for the degree of the anaerobic metabolism of any ischemic tissue. Its levels correlate with mortality in patients with AMI, but normal serum lactate levels do not exclude AMI, and vice versa, increased levels are not exclusive to AMI [11]. In combination with clinical signs and imaging findings, it should be used as additional information for diagnosis and perioperative/-interventional monitoring. Furthermore, leukocytosis is known as a non-specific predictor of an unfavorable course of AMI [12]. Measuring procalcitonin levels can be helpful for the exclusion of acute ischemia [13]. However, these parameters should not be overstated because of their lack of sensitivity and specificity.

\section{Therapeutic Approaches}

The type of therapy needs to be concordant with the radiologic findings and the patients' clinical status. Emergency surgery is demanded for unstable patients and those with signs of peritonitis [1]. If there are no signs of peritonitis or intestinal gangrene, interventional pharmacotherapy with local fibrinolysis should be considered in case of peripheral embolism. The accompanying intensive care management includes volume replacement, systemic anticoagulation and antibiotic therapy, as well as close patient monitoring to rule out secondary organ failure.

\section{Surgical Options}

Clinical signs of peritonitis, any evidence of intestinal gangrene, central occlusion of the SMA, or failure of endovascular options require an immediate surgical treatment $[1,14]$. The goal of treatment is to obtain arterial reperfusion before intestinal resection. Therefore, surgeons either need to be trained in techniques of embolectomy and reconstruction of visceral arteries or a vascular 


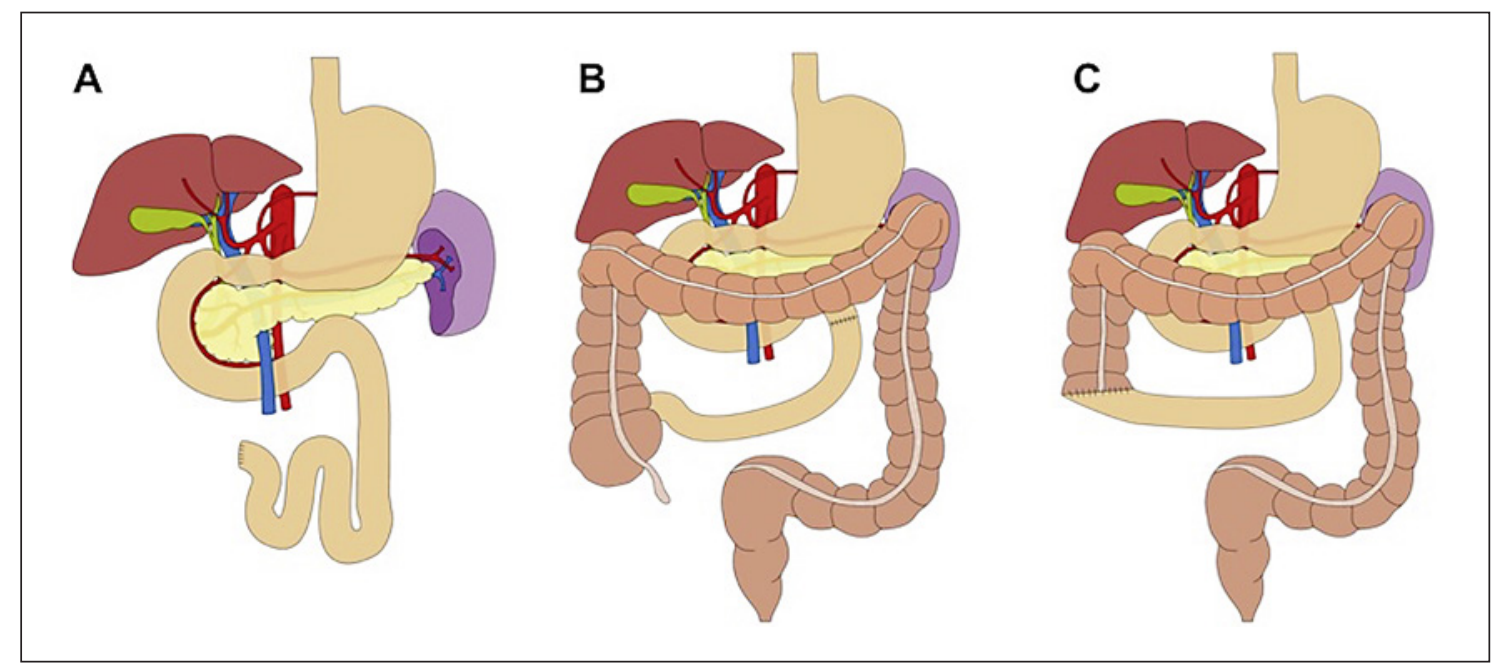

Fig. 4. Critical length of remaining small bowel after resection. A $100 \mathrm{~cm}$ in case of terminal jejunostomy (colon removed). B $65 \mathrm{~cm}$ in case of a jejuno-colonic anastomosis (colon retained). C $35 \mathrm{~cm}$ in case of a jejuno-ileal anastomosis (ileo-cecal region retained). Reproduced with kind permission from Georg Thieme Verlag KG, Stuttgart, Germany - modified from [2].

surgeon has to be consulted $[1,2,14]$. Figure 3 shows the intraoperative situs for an embolic occlusion of the SMA prior to catheter embolectomy.

In addition, the irreversibly ischemic parts of the intestine need to be resected in order to control the septic focus. The damage of the mucosal layer in many cases is much greater than estimated. Discontinuous resections with or without temporary diversion should always be considered prior to anastomosis ("damage control"). The underestimation of an ischemic segment that might lead to an anastomotic leak further contributes to a high morbidity and mortality [15]. Bowel segments with an 'uncertain' reperfusion require a scheduled "second-look" operation usually after $12 \mathrm{~h}$ to $24 \mathrm{~h}$. In case of unavoidable major bowel resections, critical residual length limits must be kept in mind [16]:

- $100 \mathrm{~cm}$ in case of a terminal jejunostomy (ileum and colon removed) (Fig. 4A).

- $65 \mathrm{~cm}$ in case of a jejuno-colonic anastomosis (colon retained) (Fig. 4B).

- $35 \mathrm{~cm}$ in case of a jejuno-ileal anastomosis (ileo-cecal region retained) (Fig. 4C).

Resection beyond these critical lengths inevitably leads to short bowel syndrome with need for permanent parenteral nutrition or small bowel transplantation $[16,17]$. The primary treatment for intestinal failure due to major bowel resections is total parenteral nutrition. Small bowel transplantation is suggested for patients who develop life-threatening complications related to parenteral nutrition administration [17]. Small intestine allografts are very immunogenic compared to other organ grafts leading to higher organ rejection rates. Therefore, higher lev- els of immunosuppression are necessary, which leads to more infectious complications compared to other solid organ transplants [17]. In order to minimize complications and achieve better patient outcomes after small bowel transplantation, these highly specialized procedures including postoperative surveillance should only be performed in experienced medical centers.

Extended resections in elderly patients with relevant co-morbidities have to be considered carefully. In a relevant proportion of these patients with AMI, surgical exploration comes too late, and palliative care has to be started [18].

\section{Endovascular Techniques}

Currently, endovascular techniques for arterial occlusion have taken on a much greater significance in cases without clinical signs of peritonitis and provided the patient is hemodynamically stable. Endovascular techniques can be considered for the diagnosis and treatment of AMI in these patients (Fig. 5). The therapeutic endovascular approach consists of angiographic catheter aspiration embolectomy and catheter lysis with recombinant tissue plasminogen activator, urokinase, or pharmacotherapy with prostaglandin E1 [19]. Fractioning of the thrombus with a guide wire can additionally increase the effect of the fibrinolytic agent. The aim is to re-open the main arterial branches of the SMA to allow the remaining occluded segments of intestine to be perfused resulting in good collateral growth [1]. If fibrinolysis and/or pharmacotherapy show changes of the vascular wall, percutaneous transluminal angioplasty (PTA) via the femoral artery can recanalize arteriosclerotic vascular occlusions or 


\section{Endovascular Therapeutic Options}

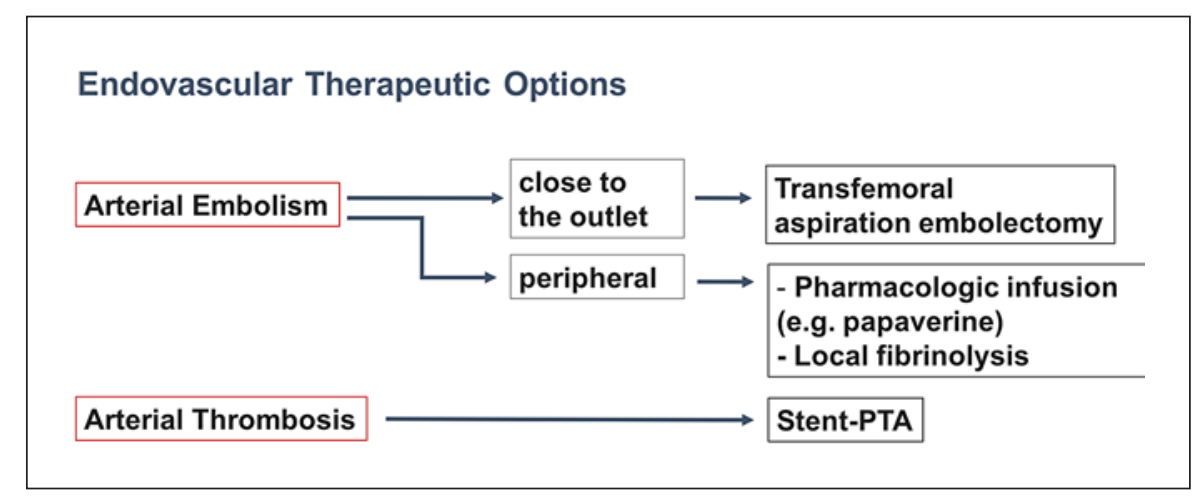

Fig. 5. Endovascular therapeutic options for hemodynamically stable patients with no signs of peritonitis.

stenoses. In case of a central thrombosis of the SMA, recanalization is secured by stent application following lysis of the clot. In combination with surgery this also allows retrograde catheterization using a peripheral SMA tributary to obtain access to the central segment [20].

\section{Intensive Care}

In addition to a prompt diagnosis and therapy, the accompanying intensive care management plays a fundamental role in the treatment of mesenteric ischemia. Immediate stabilization of hemodynamics by sufficient intravenous fluid replacement is essential in case of a systemic inflammatory response syndrome caused by volume depletion into the ischemic bowel segments. Additionally, anticoagulation needs to be started intraoperatively with a bolus application of 5,000 IE heparin followed by perfusor-controlled heparin administration [1, 6]. Systemic antibiotic therapy is mandatory and should be started as soon as possible. Toxic-ischemic end products and bacterial translocation may cause a progression of severe sepsis with secondary organ failure [1].

\section{Non-Occlusive Mesenteric Ischemia and Intestinal Venous Thrombosis}

\section{Pathophysiology and Risk Factors for NOMI}

NOMI is a pathological condition caused by vasoconstriction of splanchnic arteries without an underlying structural stenosis and is characterized by different degrees of ischemia up to gangrene in different intestinal segments. Mortality rates for NOMI has been reduced by introducing selective angiography with local application of vasodilators from 90 to $50-70 \%$ [21-24]. NOMI frequently involves the SMA territory and can lead to a severe, diffuse intestinal necrosis [22]. The compromised SMA blood supply can also comprise the proximal colon due to involvement of the ileocolic artery. The symptoms of NOMI are different from those of acute occlusive ischemia and detection often represents a challenge. Its inci- dence is especially increased in two different patient groups: in patients on chronic hemodialysis with consecutive hypovolemia and intestinal vasospasm as well as in patients after cardiac surgery including extracorporeal circulation $[1,11,22,23]$. NOMI is present in around $0.5-1 \%$ of all patients after cardiac surgery $[11,23]$. A reduced cardiac output or a perioperative hypotonic phase can lead to a vascular constriction of the splanchnic area. Heart-lung machines can additionally contribute to the reduced splanchnic blood flow [1,22-24]. Next to surgical factors such as operation time or the need for an intraaortic balloon pump, patient age, an impaired left ventricular function, peripheral vascular disease, and cerebrovascular and renal insufficiency may also contribute to the risk of developing a NOMI [1,22-24].

\section{Diagnosis and Treatment of NOMI}

Clinical examination in these patients is challenging and of limited value due to intubation and sedation. Abdominal distension, signs of sepsis, and inflammatory parameters need to be assessed carefully. Lactate serum levels are frequently increased after surgery with extracorporeal circulation; therefore, increased levels are no proof of mesenteric ischemia but can serve as an additional parameter [21-24]. For stable patients with NOMI, interventional catheter angiography is recommended. This enables diagnosis and treatment with selective application of vasodilators into the SMA and can successfully interrupt generalized vascular spasm [1]. In addition, contrast-enhanced CT plays an essential role for the diagnosis and differential diagnoses of NOMI $[1,6,22]$. CT can add further information regarding the degree of intestinal malperfusion. However, in a recent study by Myazawa et al. [22], "time from CT to injecting vasodilator" was the only factor of survival for the NOMI patients. Hence, the issue of time remains crucial, and angiography should be performed within a narrow time frame in order to improve the prognosis. Control angiography has to follow in order to confirm the success of vasodilation (Fig. 6). 
Fig. 6. NOMI after cardiac surgery before and after intra-arterial Prostavasin infusion. NOMI: non-occlusive mesenteric ischemia. Reproduced with kind permission from "Deutsches Ärzteblatt," Deutscher Ärzte-Verlag GmbH, Cologne, Germany - modified from [1].
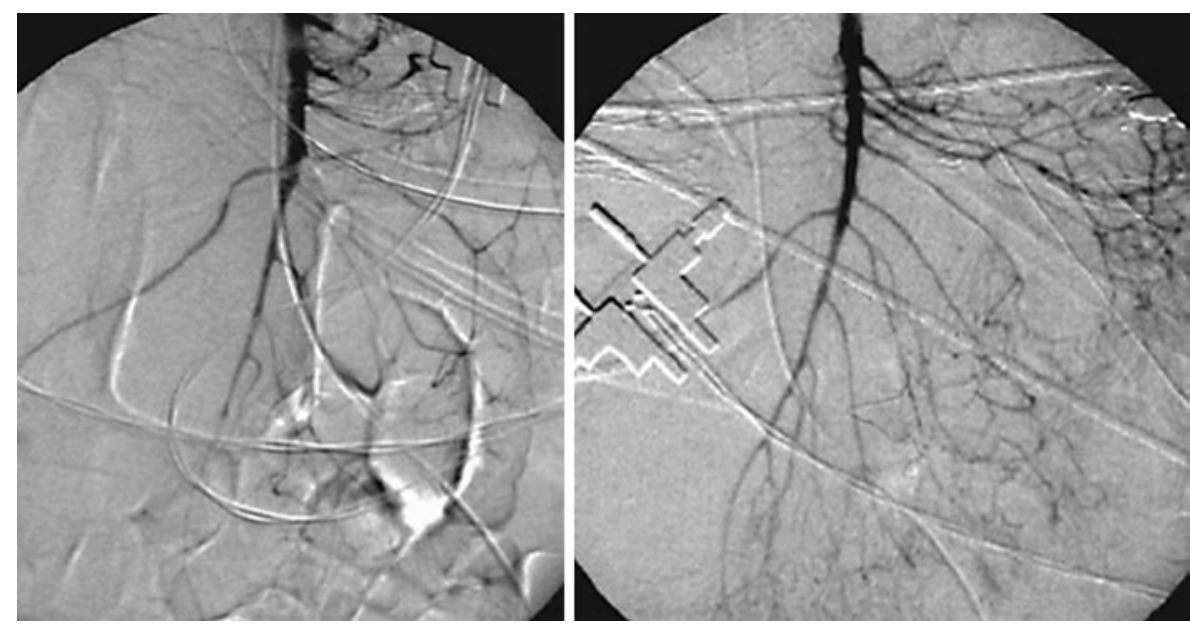

Fig. 7. Portal vein thrombosis before and 7 days after ante- and retrograde catheter lysis. reproduced with kind permission from Georg Thieme Verlag KG, Stuttgart, Germany - modified from [2].

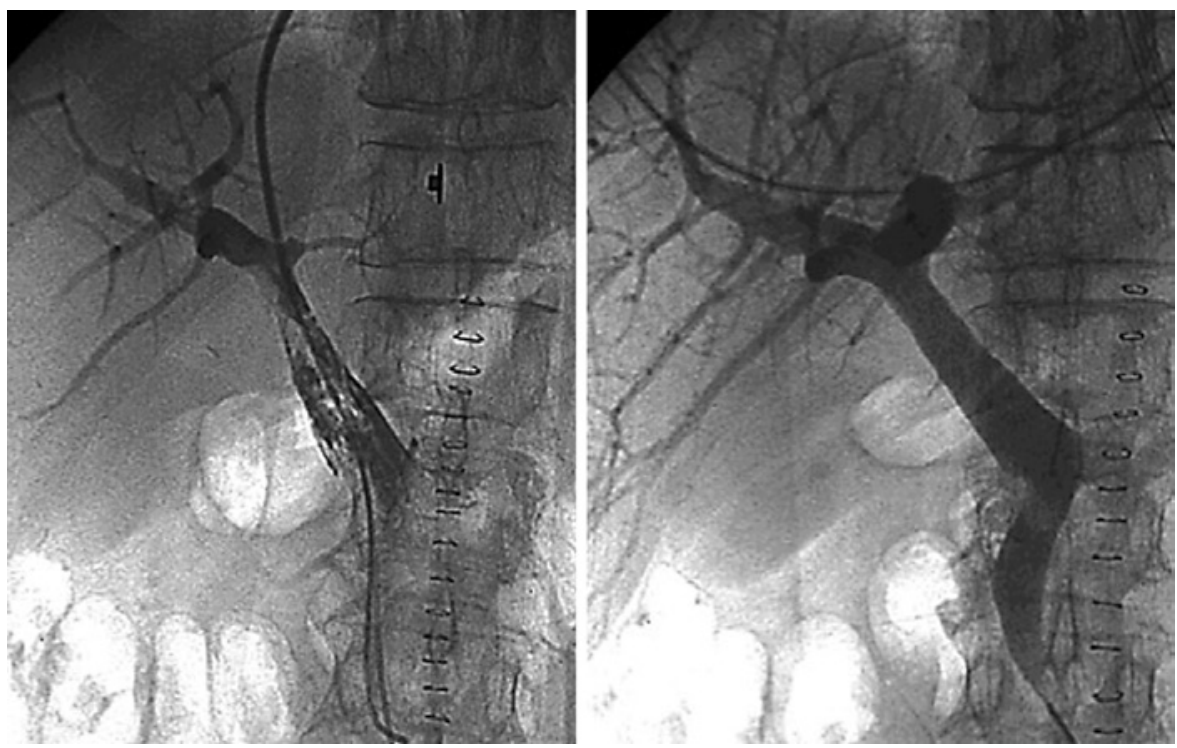

Unstable patients or those with high suspicion of intestinal gangrene require immediate surgery with abdominal exploration and resection of irreversibly damaged ischemic segments. Subsequently, these patients should receive angiographic examination with concomitant pharmacotherapy for treating the underlying vasospasm. A "second look" operation should be performed if there is any doubt about the remaining intestinal perfusion.

\section{Intestinal Venous Thrombosis}

Thrombosis might occur in case of stagnant blood flow, hypercoagulability, and vascular alterations. If centrally located and affecting several downstream areas, a venous thrombosis can lead to irreversible damage to the intestinal wall $[25,26]$. However, an isolated thrombosis of the superior mesenteric vein can be compensated by collateral flow. In contrast, an additional complete thrombosis of the portal vein is associated with venous infarction of small bowel segments [1]. Clinical symptoms are mostly less specific and depend on the extent of thrombosis. The biphasic contrast-enhanced CT is the imaging of choice for venous thrombosis, revealing alterations of the intestinal wall and ascites at the same time. Patients with peritonitis require emergency surgery with exploration and resection of infarcted segments. Antegrade transmesenteric catheter thrombolysis with the catheter inserted via a side-branch vein and placed into the thrombus can be initiated intraoperatively for subsequent local lysis: recombinant tissue plasminogen activator $(2 \mathrm{mg} / \mathrm{h})$ is administered for 2-3 days, accompanied by close angiographic controls $[1,6]$. This concept is completed by antegrade lysis via TIPS-approach postoperatively (Fig. 7). A second-look operation is recommended in patients who show extensive bowel involvement at primary exploration [4]. The treatment of choice in stable patients 
without signs of peritonitis is the recanalization via a transjugular transhepatic access, with or without transjugular intrahepatic portosystemic shunt (TIPS) placement $[26,27]$. TIPS is effective in reducing portal hypertension and recanalization of the portal vein because it allows direct access to the portal venous system where thrombolysis can be performed with subsequent TIPS placement to promote increased portal flow rate. The angiographic images of Figure 7 depict the course of a portal vein thrombosis before and 7 days after ante- and retrograde catheter lysis. In case of additional involvement of the portal vein, endovascular recanalization is recommended even in clinically compensated patients. This should even be done up to 4 weeks after the signal event in order to prevent secondary complications of portal hypertension such as esophageal or gastric varices. In case of a septic thrombosis, a systemic intravenous antibiotic treatment has to be administered since the recanalization of the portal vein can lead to the release of bacteria within the thrombus into the bloodstream [1].
In conclusion, emergency physicians need to be highly aware of the time-dependent course of AMI. Symptoms and patient-dependent risk factors should be kept in mind since prompt and targeted use of the appropriate diagnostics and interventions are the only way to reduce the persistent high mortality rates for AMI.

\section{Conflict of Interest Statement}

The authors have declared that no conflicts of interest exist.

\section{Funding Sources}

No funding was received for the present article.

\section{Author Contributions}

F.K., T.S.S. and E.K. wrote the manuscript. All authors revised and approved the manuscript for publication.

\section{References}

1 Klar E, Rahmanian PB, Bücker A, Hauenstein K, Jauch KW, Luther B. Acute mesenteric ischemia: a vascular emergency. Dtsch Arztebl Int. 2012 Apr;109(14):249-56.

2 Kuehn F, Klar E: Vaskuläre Komplikationen im Darmtrakt. Gastroenterologie Up2date 2014; 10: 159-170. https://doi.org/10.1055/ s-0034-1377490.

3 Luther B, Mamopoulos A, Lehmann C, Klar E. The Ongoing Challenge of Acute Mesenteric Ischemia. Visc Med. 2018 Jul;34(3):21723.

4 Bala M, Kashuk J, Moore EE, Kluger Y, Biffl W, Gomes CA, et al. Acute mesenteric ischemia: guidelines of the World Society of Emergency Surgery. World J Emerg Surg. 2017 Aug; 12(1):38.

5 Acosta S. Mesenteric ischemia. Curr Opin Crit Care. 2015 Apr;21(2):171-8.

6 Luther B. Intestinale Durchblutungsstörungen. Mesenterialinfarkt, Angina abdominalis, Therapieoptionen, Prognosen. Steinkopff Verlag Darmstadt; 2001. https://doi.org/ 10.1007/978-3-642-57620-1.

7 Luther B, Moussazadeh K, Müller BT, Franke C, Harms JM, Ernst S, et al. [The acute mesenteric ischemia - not understood or incurable?]. Zentralbl Chir. 2002 Aug;127(8):67484.

8 Aschoff AJ, Stuber G, Becker BW, Hoffmann $\mathrm{MH}$, Schmitz BL, Schelzig H, et al. Evaluation of acute mesenteric ischemia: accuracy of biphasic mesenteric multi-detector CT angiography. Abdom Imaging. 2009 May-Jun;34(3) 345-57.

9 Yang H, Wang BL. Evaluation of the diagnostic value of multi-slice spiral CT in acute mesenteric ischemic diseases: a meta-analysis of randomized controlled trials. Eur Rev Med Pharmacol Sci. 2019 Dec;23(23):10218-25.

10 Kellow ZS, MacInnes M, Kurzencwyg D, Rawal S, Jaffer R, Kovacina B, et al. The role of abdominal radiography in the evaluation of the nontrauma emergency patient. Radiology. 2008 Sep;248(3):887-93.

11 Filsoufi F, Rahmanian PB, Castillo JG, Scurlock C, Legnani PE, Adams DH. Predictors and outcome of gastrointestinal complications in patients undergoing cardiac surgery. Ann Surg. 2007 Aug;246(2):323-9.

12 Block T, Nilsson TK, Björck M, Acosta S. Diagnostic accuracy of plasma biomarkers for intestinal ischaemia. Scand J Clin Lab Invest. 2008;68(3):242-8.

13 Markogiannakis $\mathrm{H}$, Memos N, Messaris E, Dardamanis D, Larentzakis A, Papanikolaou $\mathrm{D}$, et al. Predictive value of procalcitonin for bowel ischemia and necrosis in bowel obstruction. Surgery. 2011 Mar;149(3):394-403.

14 Duran M, Pohl E, Grabitz K, Schelzig H, Sagban TA, Simon F. The importance of open emergency surgery in the treatment of acute mesenteric ischemia. World J Emerg Surg. 2015 Sep;10(1):45.

15 Unalp HR, Atahan K, Kamer E, Yaşa H, Tarcan E, Onal MA. [Prognostic factors for hospital mortality in patients with acute mesenteric ischemia who undergo intestinal resection due to necrosis]. Ulus Travma Acil Cerrahi Derg. 2010 Jan;16(1):63-70.

16 Messing B, Crenn P, Beau P, Boutron-Ruault MC, Rambaud JC, Matuchansky C. Longterm survival and parenteral nutrition dependence in adult patients with the short bowel syndrome. Gastroenterology. 1999 Nov;117 (5):1043-50.

17 Kesseli S, Sudan D. Small Bowel Transplantation. Surg Clin North Am. 2019 Feb;99(1): 103-16.

18 Ritz JP, Germer CT, Buhr HJ. Prognostic factors for mesenteric infarction: multivariate analysis of 187 patients with regard to patient age. Ann Vasc Surg. 2005 May;19(3): 328-34.
19 Schoots IG, Levi MM, Reekers JA, Lameris JS van Gulik TM. Thrombolytic therapy for acute superior mesenteric artery occlusion. J Vasc Interv Radiol. 2005 Mar;16(3):317-29.

20 Pisimisis GT, Oderich GS. Technique of hybrid retrograde superior mesenteric artery stent placement for acute-on-chronic mesenteric ischemia. Ann Vasc Surg. 2011 Jan; 25(1):132.e7-11.

21 Björck M, Wanhainen A. Nonocclusive mesenteric hypoperfusion syndromes: recognition and treatment. Semin Vasc Surg. 2010 Mar;23(1):54-64.

22 Miyazawa R, Kamo M. What affects the prognosis of NOMI patients? Analysis of clinical data and CT findings. Surg Endosc. 2019 Dec; Epub ahead of print. https://doi.org/10.1007/ s00464-019-07321-9.

23 Mangi AA, Christison-Lagay ER, Torchiana DF, Warshaw AL, Berger DL. Gastrointestinal complications in patients undergoing heart operation: an analysis of 8709 consecutive cardiac surgical patients. Ann Surg. 2005 Jun;241(6):895-901.

24 Lim JY, Kim JB, Jung SH, Choo SJ, Chung $\mathrm{CH}$, Lee JW. Risk factor analysis for nonocclusive mesenteric ischemia following cardiac surgery: A case-control study. Medicine (Baltimore). 2017 Sep;96(37):e8029.

25 Harnik IG, Brandt LJ. Mesenteric venous thrombosis. Vasc Med. 2010 Oct;15(5):407-18.

26 Goykhman Y, Ben-Haim M, Rosen G, Carmiel-Haggai M, Oren R, Nakache R, et al. Transjugular intrahepatic portosystemic shunt: current indications, patient selection and results. Isr Med Assoc J. 2010 Nov;12(11): 687-91.

27 Intagliata NM, Caldwell SH, Tripodi A. Diagnosis, Development, and Treatment of Portal Vein Thrombosis in Patients With and Without Cirrhosis. Gastroenterology. 2019 May; 156(6):1582-1599.e1. 